\title{
ORIGINAL
}

\section{INCIDENCIA DE LAS NEUMONIAS NEUMOCÓCICAS EN EL ÁMBITO HOSPITALARIO EN LA COMUNIDAD VALENCIANA DURANTE EL PERIODO 1995-2001}

Ana María Comes Castellano, José Antonio Lluch Rodrigo, Antonio Portero Alonso, Eliseo Pastor
Villalba y Miguel Sanz Valero

Centro Consultor en Vacunología del Centro Superior de Investigaciones en Salud Pública. Dirección General de Salud Pública, CSISP. Consellería de Sanidad de Valencia.

\section{RESUMEN}

Fundamento: Las neumonías neumocóccicas son un importante problema de salud. El objetivo del estudio es conocer la evolución en la Comunidad Valenciana de las neumonías neumocóccicas hospitalarias desde 1995 a 2001.

Métodos: Del Conjunto Mínimo Básico de Datos se seleccionaron los casos de neumonías neumocócica en la Comunidad Valenciana. Se calculó el intervalo de confianza (95\%) y ANOVA con el programa SPSS, así como la incidencia y su evolución en el tiempo y las interacciones con las variables de sexo, edad, y provincia. Se calculó igualmente el promedio de estancia hospitalaria.

Resultados: La incidencia del periodo fue de 29 casos por 100.000. Disminuye desde 1996 y se estabiliza en los últimos años del estudio. En personas mayores de 65 años la incidencia fue de 101 por 100.000 , mientras que en menores de 5 años fue de 76 por 100.000. La incidencia en mujeres fue de 22 por 100.000 y de 37 por 100.000 en hombres. La incidencia por provincias fue de 31 para Valencia, 21 para Alicante y 24 para Castellón; en Valencia y Alicante tienden a estabilizarse, mientras que ascienden en Castellón. La estancia media fue de 10,6 \pm 9 días, siendo de 12,5 en el grupo de 45 a 64 años.

Conclusiones: Las neumonías neumocócicas son más frecuentes en las edades extremas. Afectan más a hombres, especialmente en mayores de 65 años. Se observa una evolución diferente en la provincia de Castellón. El promedio de estancia varía según la edad, sin existir diferencias significativas entre sexo o grupo de edad.

Palabras clave: Neumonía neumocócica. Streptococcus pneumoniae. Incidencia. Estancia media. Hospitalización.

Correspondencia:

José Antonio Lluch Rodrigo

C/ Micer Mascó, 31 y 33

46010 Valencia

Correo electrónico: 1luch_jos@gva.es
ABSTRACT

Incidence of Pneumococcal Pneumonias in Hospital Settings in the Autonomous Community of Valencia throughout the 1995-2001 Period. Spain

Background: Pneumococcal pneumonias comprise a major health problem. This study is aimed at ascertaining the evolution of pneumococcal pneumonias in hospital settings within the Autonomous Community of Valencia throughout the 1995-2001 period.

Methods: The cases of pneumococcal pneumonias were selected from the Minimum Basic Data Set. The confidence interval $(95 \%)$ and ANOVA were calculated using the SPSS program, the incidence and evolution over the course of time, in addition to the interactions with the gender, age and province variables were calculated. The average length of hospital stay was also calculated.

Results: The incidence for the period under study was that of 29 cases/100,000, having dropped since 1996 and stabilizing during the final years of the study. Among individuals over age 65, the incidence was that of 101/100,000, whilst among those under five years of age, it was $76 / 100,000$. The incidence among females was $22 / 100,000$ and $37 / 100,000$ among males. The incidence by provinces was 31 for Valencia, 21 for Alicante and 24 for Castellón; tending to stabilize in Valencia and Alicante, while rising in Castellón. The average length of stay was $10.6+9$ days, being 12.5 days for the 45 64 age range.

Conclusions: Pneumococcal pneumonias are more frequent among either the very young or very old and affect males more, especially those over age 65 . A different evolution was found in the province of Castellón. The average length of stay varies according to age, there being no significant difference between genders or age groups.

Key words: Pneumococcal infections. Streptococcus pneumoniae. Incidence. Length of stay. Hospitalization. 


\section{INTRODUCCIÓN}

El Streptococcus pneumoniae ocasiona enfermedades invasivas y no invasivas. Si bien éstas últimas son más frecuentes las primeras causan cuadros clínicos más graves, como bacteriemia o meningitis. El neumococo es una de las primeras causas de infecciones graves adquiridas en la comuni$\mathrm{dad}^{1}$. El riesgo es mayor en las edades extremas de la vida y cuando coexisten otros factores de riesgo (tabaquismo, enfermedades crónicas) ${ }^{2}$. La neumonía es la causa de muerte más frecuente por infección neumocócica, con una letalidad de entre el 2 y el $16 \% \%^{3,4,5}$

En la Comunidad Valenciana (CV) la incidencia de neumonía por cualquier causa se ha estimado alrededor del 200 por 100.000 habitantes, variando en función de factores como la edad o el género ${ }^{6}$. La mortalidad en nuestro entorno es algo mayor del 0,40 por $100.000^{7}$, lo que supone el $2,65 \%$ de todas las muertes.

El hecho de que la morbilidad y mortalidad sea superior en personas mayores de 64 años ${ }^{10}$, con incidencias de neumonías neumocócicas de entre el 3 y el 8 por $1000^{11}$ y letalidad del $12,38 \%{ }^{12}$, hace pensar que esta situación, así como el impacto económico que supone la enfermedad, pueden agravarse en un futuro por el envejecimiento de la población y por la aparición de resistencias frente a los antibióticos ${ }^{8}$.

El planteamiento de este artículo nace de la recomendación dentro del Plan de Vacunación del Neumococo en la CV de vacunar sistemáticamente a la población mayor de 64 años, y para poder calcular el efecto de esta medida en la población de riesgo.

El objetivo de este estudio es analizar la magnitud y la evolución de la incidencia de neumonía neumocócica en los hospitales públicos de la $\mathrm{CV}$ durante un periodo de siete años.

\section{MATERIAL Y MÉTODOS}

Los datos fueron tomados del Conjunto Mínimo Básico de Datos (CMBD) de los hospitales públicos de la CV desde el año 1995 hasta 2001, facilitados por el Servicio de Análisis de la Actividad Asistencial de la Consellería de Sanitat de Valencia, que valida los datos antes de su difusión. Se han seleccionado los casos diagnosticados según la CIE-9, como 481 (neumonía neumocócica) en alguno de los campos diagnósticos. Se analizó la incidencia en función de las variables edad, género, provincia de residencia y se calcularon los promedios de estancia para todo el periodo y para cada año, observando su evolución en el tiempo. Los grupos de edad definidos fueron G1, población entre 0-5 años; G2, población de 6-14 años; G3, población de 15-45 años; G4, población de 46-64 años, y G5 mayores de 64 años. Los denominadores poblacionales para calcular la incidencia se obtuvieron del Instituto Nacional de Estadística ${ }^{9}$. Se han comparado los resultados con los obtenidos en análisis similares realizados para el conjunto de neumonías. Se han estudiado estadísticas descriptivas, comparación de medias y ANOVA utilizando el programa SPSS, versión 11.0 para Windows. Para la comparación de la incidencia se han calculado los intervalos de confianza al 95\% (IC $95 \%)$.

\section{RESULTADOS}

Los casos del CMBD con diagnostico 481 (neumonía neumocócica) en el periodo de estudio fueron 8.428 , el $14,1 \%$ de todas las neumonías hospitalizadas. La incidencia global fue de 29 casos por 100.000 habitantes, con un IC 95\% $\pm 0,63$. La incidencia global aumenta en el año 1996, para disminuir posteriormente y estabilizarse en los años 2000 y 2001 (tabla 1, figura 1). Por edades: el $14,2 \%$ de los casos se dio en menores de 5 años, el 3,4\% en el grupo de 6 a 14 años, el $12,7 \%$ en el grupo de 15 a 45 años, el 14,4\% 
INCIDENCIA DE LAS NEUMONIAS NEUMOCÓCICAS EN EL ÁMBITO HOSPITALARIO EN LA COMUNIDAD VALENCIANA...

Tabla 1

Tasas de incidencia por $\mathbf{1 0 0 . 0 0 0}$ de la Neumonía neumocócica, por grupos de edad y año

\begin{tabular}{|c|c|c|c|c|c|c|c|c|}
\hline Grupos de Edad & $\mathbf{1 9 9 5}$ & $\mathbf{1 9 9 6}$ & $\mathbf{1 9 9 7}$ & $\mathbf{1 9 9 8}$ & $\mathbf{1 9 9 9}$ & $\mathbf{2 0 0 0}$ & $\mathbf{2 0 0 1}$ & Global \\
\hline $0-5$ años & 94 & 99 & 78 & 71 & 56 & 64 & 69 & 76 \\
\hline $6-14$ años & 6 & 34 & 10 & 9 & 3 & 11 & 14 & 10 \\
\hline $15-45$ años & 8 & 10 & 9 & 9 & 8 & 6 & 7 & 8 \\
\hline $46-64$ años & 20 & 23 & 19 & 22 & 18 & 16 & 17 & 19 \\
\hline$\geq 65$ años & 97 & 121 & 109 & 110 & 105 & 81 & 87 & 101 \\
\hline Total & 29 & 36 & 31 & 31 & 28 & 24 & 26 & 29 \\
\hline
\end{tabular}

Figura 1

Incidencias de la neumonía neumocócica por año, por género y globales

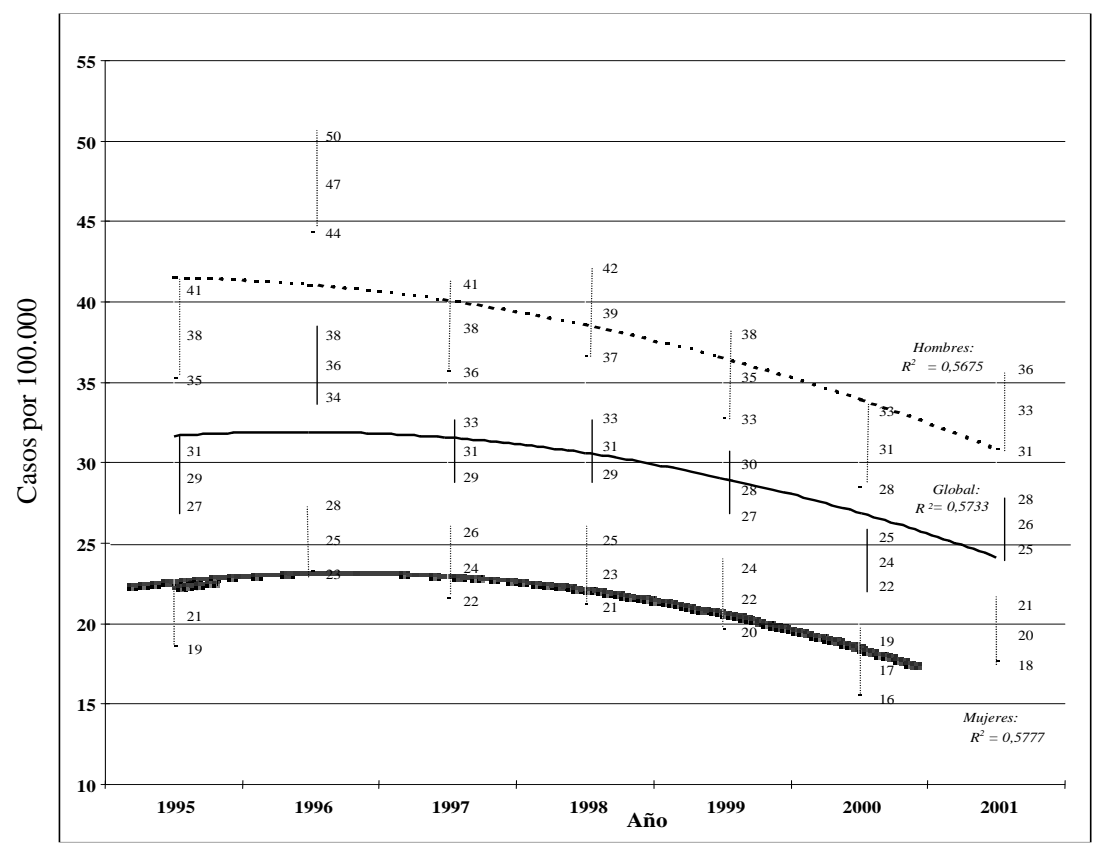

$R^{2}$ : Coeficiente de correlación. 
Tabla 2

Incidencias e intervalos de confianza globales para todos los casos y por años. Incidencia e IC (intervalo de confianza) para el grupo de mayores de 64 años

\begin{tabular}{|c|c|c|c|c|c|c|}
\hline \multirow{2}{*}{ Año } & \multicolumn{3}{|c|}{ Incidencias todas las edades } & \multicolumn{3}{c|}{ Incidencias mayores de 64 } \\
\cline { 2 - 7 } & Hombres & Mujeres & Global & Hombres & Mujeres & Global \\
\hline 1995 & $38 \pm 2,74$ & $21 \pm 1,97$ & $29 \pm 1,68$ & $147 \pm 7,79$ & $60 \pm 8,07$ & $97 \pm 7,79$ \\
\hline 1996 & $48 \pm 3,06$ & $25 \pm 2,19$ & $36 \pm 1,87$ & $187 \pm 16,64$ & $72 \pm 8,86$ & $121 \pm 8,69$ \\
\hline 1997 & $39 \pm 2,76$ & $24 \pm 2,12$ & $31 \pm 1,73$ & $151 \pm 14,78$ & $79 \pm 9,15$ & $108 \pm 8,18$ \\
\hline 1998 & $40 \pm 2,79$ & $23 \pm 2,09$ & $31 \pm 1,74$ & $152 \pm 14,69$ & $79 \pm 9,08$ & $110 \pm 8,13$ \\
\hline 1999 & $36 \pm 2,62$ & $22 \pm 2,01$ & $29 \pm 1,64$ & $143 \pm 13,92$ & $77 \pm 8,78$ & $105 \pm 7,78$ \\
\hline 2000 & $40 \pm 2,42$ & $17 \pm 1,78$ & $24 \pm 1,49$ & $118 \pm 12,49$ & $54 \pm 7,29$ & $81 \pm 6,77$ \\
\hline 2001 & $33 \pm 2,42$ & $19 \pm 1,87$ & $26 \pm 1,55$ & $130 \pm 12,9$ & $54 \pm 7,18$ & $87 \pm 6,88$ \\
\hline Global & $37 \pm 1,02$ & $22 \pm 0,76$ & $29 \pm 0.63$ & $146 \pm 5,39$ & $68 \pm 3,15$ & $101 \pm 2,92$ \\
\hline
\end{tabular}

Tasas por 100.000

en los de 46 a 64 años y el 54,4\% restantes, para los mayores de 64 años. La media de edad fue de 55,59 años con una desviación estándar (DS) de 29,42, destacando una media de edad en el grupo de mayores de 64 años de 77,63 años (DS: 7,45) y dentro de este grupo de 76,84 en hombres (DS 7,16) y 78,88 en mujeres (DS: 7,72). La incidencia más elevada se dio en los mayores de 64 años y en menores de 5 años (tabla 1). Respecto del total de neumonías, en mayores de 64 años la neumonía neumocócica representa un $13,6 \%$ para el periodo analizado, oscilando entre el $19,5 \%$ del año 1996 y el $10 \%$ de 2001 (figura 2). Por géneros: El 62,5\% eran hombres (5.223 casos) y el $37,4 \%$ mujeres (3.133). La razón hombre/mujer fue de 1,7 para el periodo, siendo de 1,59 en mayores de 64 años. La incidencia de la neumonía neumocócica fue para el periodo de $37 \pm 1,02$ por 100.000 en hombres y $22 \pm 0,76$ por 100.000 en mujeres (figura 1). Se observa que la incidencia es aproximadamente dos veces mayor en hombres que en mujeres. Estas diferencias son estadísticamente significativas para todos los grupos de edad excepto para el grupo de 0 a 14 años, con incidencias de 11 por 100.000 frente a 5 por 100.000 en el grupo de 15 a 45 años y de 27 por 100.000 frente a 11 por 100.000 en el grupo de 46 a 64 años la incidencia en hombres es de 27 por 100.000 y de 11 por 100.000 en mujeres. Estas diferencias son más acusadas en mayores de 64 años, donde la incidencia es de 146/100.000 en hombres y 68/100.000 en mujeres (tabla 2). Por provincias: El 55,7\% de los casos se registraron en la provincia de Valencia, el 25,1\% en Alicante y el 9,2\% en Castellón, y hubo un $10 \%$ de casos perdidos por errores. La incidencia fue de $31 \pm 0,87 / 100.000$, para Valencia $(38 \pm 1,40$ para hombres y de $23 \pm 1,06$ para 
Figura 2

Incidencia de la neumonía neumocócica, en el grupo de mayores de 64 años, frente a la incidencia de todas las neumonías

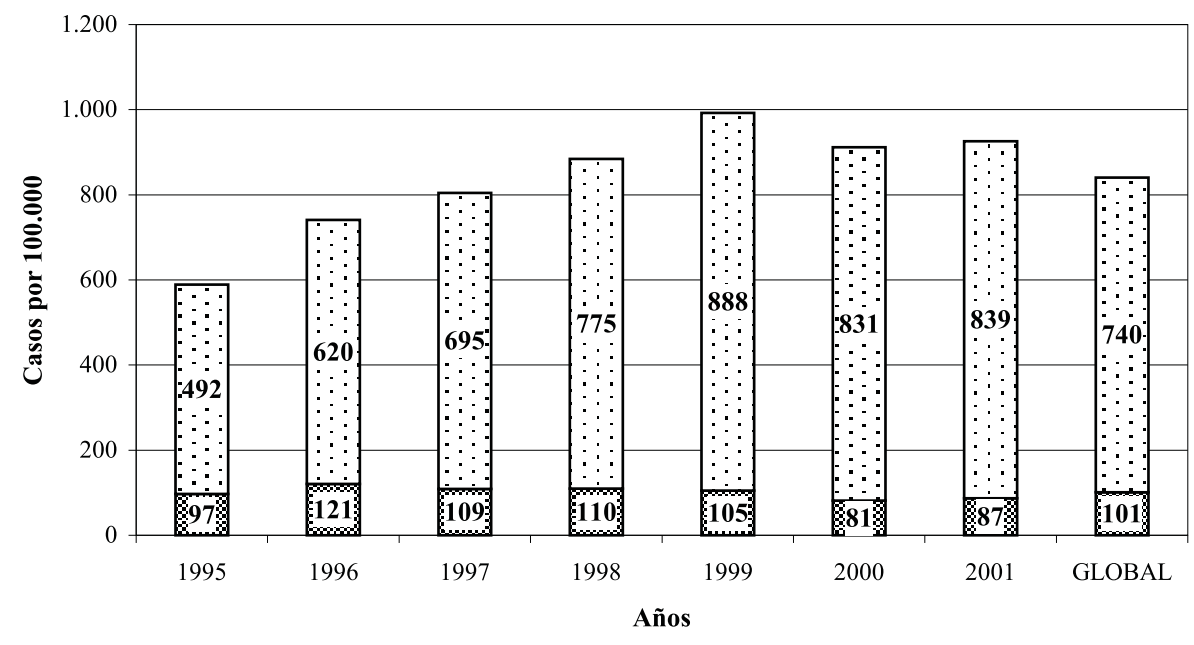

冈 Neumonías neumococcicas

$\square$ Todas las causas

mujeres). En Alicante la incidencia fue de $21 \pm 0,91 / 100.00$, (IC 95\%28 1 1,49, para hombres y de $15 \pm 1,06$, para mujeres). En Castellón la incidencia fue de $24 \pm 1,67$ /

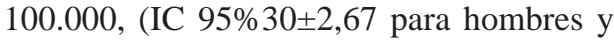
de $18 \pm 2,02$ para mujeres). Cuando se analizan los datos globales del periodo las diferencias entre la incidencia en la provincia de Valencia son estadísticamente significativas respecto a las provincias de Castellón y Alicante (figura 3). En Valencia la edad media fue de 55,8 años (DS:. 29,36). En Alicante de 53,8 años (DS: 29,88). En Castellón la edad media fue de 60,3 años (DS: 27,55). En mayores de 64 años, en Valencia, la edad media fue de 77,8 años (DS: 7,51) de 76,8 en hombres y 79,1 en mujeres, (DS: 7,23 y 7,71 respectivamente). En Alicante la edad media fue de 77,3 años (DS: 7,37) de 76,6 años en hombres y 78,5 en mujeres, (DS: 7,0 y 7,88 respectivamente). En Castellón fue de 77,93 años (DS: 7,31) de 77,5 en hombres y 78,7 años en mujeres, (DS: 7,24 y 7,39 respectivamente). La evolución difiere en las tres provincias con una tendencia a disminuir en las provincias de Alicante y Valencia hacia final del periodo de estudio, frente a un incremento mantenido en la incidencia en la provincia de Castellón. Siendo las incidencias en los tres últimos años del periodo en Valencia de 32, 23 y 24 casos por 100.000 , en Alicante de 17, 16 y 17 casos por 100.000 y para Castellón de 25, 38 y 44 casos por 100.000 (figura 5). Promedio de estancia: En los hospitales de la CV la estancia media por neumonía neumocócica es de 10,6 $\pm 9,4$ días (con un mínimo de un día, un máximo de 142 , y una mediana de 8 ), oscilando, entre 6,3 días en menores de 5 años y 12,5 días en la población entre 46-64 años, no mostrando diferencias estadísticamente significativas entre las variables estudiadas en cuanto a la duración del ingreso (tabla 3). Las personas mayores de 64 años generaron 52.547 estancias durante el periodo, siete veces más que los menores de 5 años. La población entre 46 y 64 años es la que posee el promedio de estancia mayor con 12,5 días de estancia media. No se observaron diferencias significativas entre las estancias medias generadas 
Tabla 3

Número de estancias generadas por años. Estancias medias calculadas para el período y por años, separando géneros y por grupos de edad

\begin{tabular}{|c|c|c|c|c|c|c|c|c|c|}
\hline \multirow[b]{2}{*}{ Año } & \multirow{2}{*}{$\begin{array}{l}\text { Número de } \\
\text { estancias } \\
\text { (días) }\end{array}$} & \multicolumn{2}{|c|}{ Género } & \multicolumn{5}{|c|}{ Estancias medias por grupo de edad } & \multirow{2}{*}{$\stackrel{\text { Total }}{(\bar{X} \text { días })}$} \\
\hline & & $\begin{array}{l}\text { Hombres } \\
\bar{X} \text { días(DE) }\end{array}$ & $\begin{array}{c}\text { Mujeres } \\
\bar{X} \text { días(DE) }\end{array}$ & $\begin{array}{c}0-5 \\
\text { años }\end{array}$ & $\begin{array}{l}\text { 6-14 } \\
\text { años }\end{array}$ & $\begin{array}{l}1444 \\
\text { años }\end{array}$ & $\begin{array}{l}45-64 \\
\text { años }\end{array}$ & $\begin{array}{l}\geq 65 \\
\text { años }\end{array}$ & \\
\hline 1995 & 13.145 & $11,5(9,8)$ & $10,6(9,0)$ & 5,9 & 5,5 & 12,0 & 13,5 & 12,5 & 11,2 \\
\hline 1996 & 14.756 & $10,1(8,5)$ & $10,0(10,2)$ & 5,5 & 5,3 & 10,4 & 12,5 & 11,2 & 11,7 \\
\hline 1997 & 13.389 & $10,4(8,5)$ & $11,0(8,6)$ & 5,5 & 6,0 & 9,5 & 11,8 & 11,9 & 11,2 \\
\hline 1998 & 13.628 & $11,0(9,2)$ & $103(8,4)$ & 5,9 & 7,0 & 10,7 & 12,4 & 11,6 & 11,0 \\
\hline 1999 & 12.565 & $11,3(9,9)$ & $10,0(7,6)$ & 7,2 & 11,6 & 10,3 & 12,0 & 11,2 & 10,5 \\
\hline 2000 & 10.569 & $10,9(10,9)$ & $10,3(10,2)$ & 7,3 & 7,0 & 11,9 & 12,1 & 11,2 & 10,3 \\
\hline 2001 & 11.219 & $10,3(9,9)$ & $9,8(9,4)$ & 6,1 & 7,8 & 10,7 & 13,0 & 10,5 & 10,1 \\
\hline Total & 89.236 & $10,29(9,0)$ & $10,3(9,0)$ & 6,3 & 6,6 & 10,7 & 12,5 & 11,5 & 10,6 \\
\hline
\end{tabular}

DE: desviación estándar. $\bar{X}$ : Media

por hombres o mujeres, aunque son los hombres los que generan mayor número de estancias (1,75 veces más). Los menores de 5 años, tienen un promedio de estancia más bajo (tabla 3).

\section{DISCUSIÓN}

La incidencia global encontrada para todo el periodo fue de 29 por 100.000 , similar a la de 26,2 encontrada en Cataluña ${ }^{13}$ o en EEUU $^{14,15}$, y superior a las incidencias de 4,7 por 100.000 en Finlandia ${ }^{16}$ o de 1,6 por 100.000 en Suiza ${ }^{17}$. En EEUU, la incidencia de enfermedad invasora por neumococo fue en 1998 de 24 casos por 100.000 para todas las edades y de 61 por 100.000 para los mayores de 65 años $^{14,18}$, cifras ligeramente inferiores a las encontradas en este estudio para el mismos año. Fedson et al. aportan unos datos de entre 50 y 60 casos por 100.000 para las personas mayores de $65 \mathrm{y}$ de entre 27 y 62 casos por 100.000 en población general en países como Finlandia,
Dinamarca, Israel, Australia, Inglaterra, Gales, Suecia y Noruega ${ }^{19,20}$, coincidiendo con las incidencias aportadas por otros autores $^{19-22}$. En nuestro estudio los sujetos mayores de 64 años presentaron una incidencia de 101 casos por 100.000 (rango entre $81 \mathrm{y}$ 121). Las variaciones en la incidencia podrían deberse a las diferencias en los criterios de diagnóstico, a la variabilidad en la práctica médica en diferentes países o a la monitorización de los casos ${ }^{13,23,24}$. Remarcar que si las neumonías neumocócicas con aislamiento del Neumococo representaron el $14,1 \%$ del total de neumonías, es evidente que la carga que supone esta forma clínica de la enfermedad es muy superior a la estimación que a partir del CMBD hemos hecho tanto nosotros como otros autores, que las sitúan entre el 10-25\% del total de neumonías ${ }^{2,20}$.

La razón entre hombres y mujeres, siempre mayor que 1 , coincide con los datos reseñados por otros autores ${ }^{16,25-27}$. La diferente prevalencia de factores de riesgo que se han asociado a la aparición de la neumonía, 


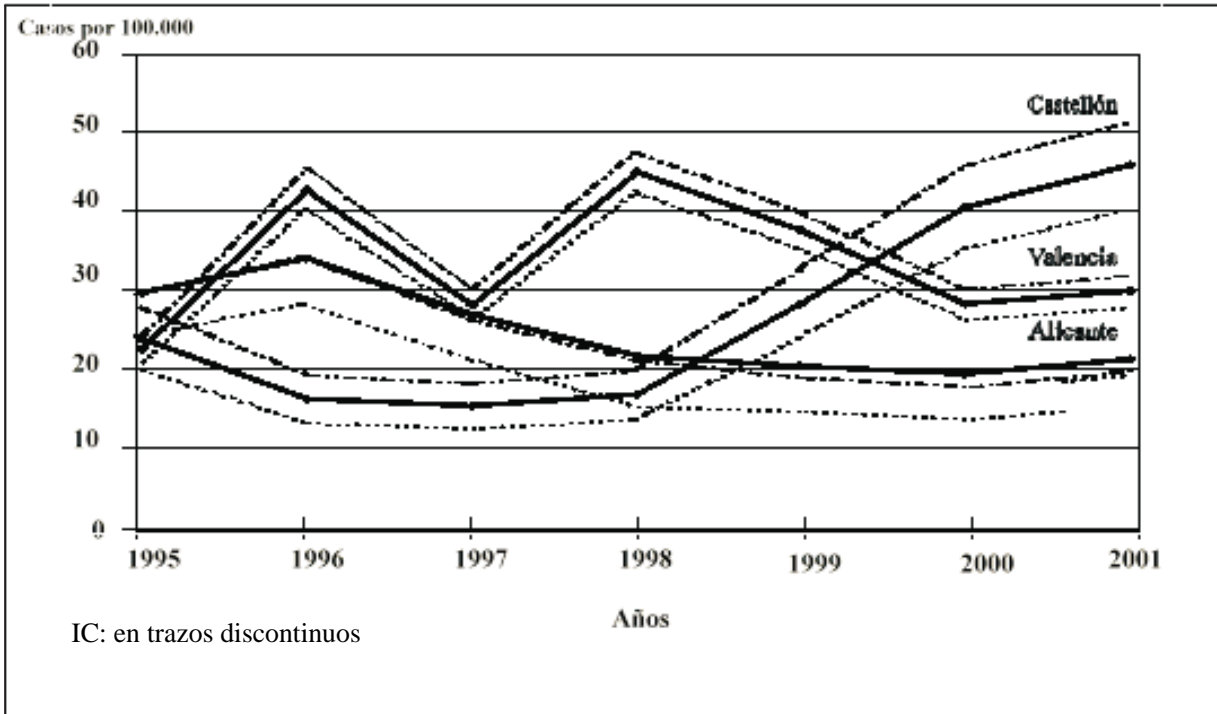

como el consumo de tabaco, podrían explicar estas diferencias. Los resultados por grupos de edad coinciden con los encontrados por otros autores ${ }^{6,28-30}$. La incidencia de la neumonía neumocócica en mayores de 64 años tiende a disminuir y estabilizarse en los últimos años del estudio.

Desde el punto de vista territorial y de forma global para todo el periodo la incidencia es sensiblemente superior en la provincia de Valencia frente a las otras dos. Sin embargo, el análisis de la evolución muestra diferencias en otros sentidos. Así mientras que en Valencia la incidencia desciende bruscamente en el año 2000, manteniéndose en el año siguiente, en Alicante la situación es estable y en Castellón se incrementa de forma progresiva, superando de forma significativa la incidencia de las otras provincias. Esta situación sólo podría explicarse por diferencias en la práctica médica, ya que no existen aparentemente otras circunstancias que pueda explicarla. Estudios posteriores podrían aclarar mejor cual es la causa de estas diferencias.
Respecto a las estancias medias en los hospitales por afección neumocócica no existen diferencias significativas referidas al género, aunque las medias de estancias son más elevadas en hombres. Se concluye, que los menores de 15 años tienen las estancias medias más cortas y que el grupo de entre 45-64 años tiene las estancias medias más largas. Los mayores de 64 años generan mayor número de estancias por año. Respecto al total de las neumonías no se concluye que la permanencia en los hospitales sea diferente dependiendo del tipo de neumonía pues no se dan diferencias estadísticamente significativas.

La incidencia por edades y su diferencia entre distintos grupos de edad podría deberse a factores ambientales, hábitos de vida u otras razones intrínsecas a los modos de vida de la población a partir de la adolescencia, así como a diferencias en el sistema inmunitario en las edades extremas de la vida. Estos factores podrían explicar la diferencia entre incidencia (y razón de casos) en hombres y mujeres a partir de cierta edad, hecho que no se percibe en la población infantil. 
La dificultad para conocer la magnitud de la morbimortalidad en la infección neumocócica, radica en la dificultad del diagnóstico y en la intensidad con que se utilicen los medios disponibles, que explicarían en parte las diferencias en la importancia relativa de los distintos serotipos en distintas localizaciones geográficas ${ }^{31}$.

Con los datos encontrados en este estudio parece razonable seguir recomendado la vacunación en las personas mayores de 64 años, a pesar de la controversia existente en cuanto a la efectividad de las vacunas disponibles. El gran número de casos así como el elevado coste que supone atenderlos justificaría la inversión.

\section{BIBLIOGRAFÍA}

1. Macfarlane JT, Finch RG, Ward MJ, Macrae AD. Hospital study of adult community-acquired pneumonia. Lancet 1982; Jul 31; 2(8292): 255-8

2. Musher D. Streptococcus pneumoniae. En: Mandell GL, Bennett JE \& Dolin R, Editores. Principles and Practice of infectious Diseases, 5th ed. Philadelphia: Churchill Livingstone; 2000.p.2128-46.

3. Ashton CM, Petersen NJ, Souchek J, Menke TJ, Yu $\mathrm{HJ}$, Pites K et al. Geographic variation in utilization rates in veterans affairs hospitals and clinics. $\mathrm{N}$ Engl J Med 1999; 340:32-9.

4. Almirall J, Casado M, Valls F, Morato I, Agudo A, Priu R et al. Estudio prospectivo de las neumonías extrahospitalarias atendidas en un hospital general. Error diagnóstico. Med Clin (Barc) 1991; 97:2504.

5. World Health Organization. Pneumococcal vaccines. Wkly Epidemiol Rec 1999; 74:177-84

6. Vacuna contra el Neumococo. Guía Práctica para Profesionales Sanitarios. Valencia: Generalitat Valenciana; 2001. Monografía Sanitaria núm 34.

7. Registro de mortalidad de la CV. Área de Epidemiología. Dirección General para la Salud Pública. Conselleria de Sanitat. (citado el: 01.05.2003). Disponible en: http://dgsp.san.gva.es/isum/ Direct.jsp?ISUM_Shortcut=MORT_ESTADIST
8. Centers for Disease Control and Prevention. Defining the public health impact of drug-resistant Streptococcus pneumoniae: report a working group. MMWR 1996; 45:1-20.

9. Instituto Nacional de Estadística. (citado:10.04.2003). Disponible en: www.INE.es

10. Sleeman K, Knox K, George R, Miller E, Waight P, Griffiths D et al. Invasive pneumococcal disease in England and Wales: vaccination implications. J Infect Dis 2001; 183: 239-46.

11. Almirall J, Morato I, Riera F, Verdaguer A, Priu R, Coll $\mathrm{P}$ et al. Incidence of community-acquired pneumonia and Chlamydia pneumoniae infection: a prospective multicentre study. Eur Respir J 1993; $6: 14-8$.

12. Fine MJ, Smith MA, Carson CA, Mutha SS, Sankey SS, Weissfeld LA et al. Prognosis and outcomes of patients with community-acquired pneumonia. A meta-analysis. JAMA 1996;275:134-41.

13. Domínguez A, Salleras L, Cardeñosa N, Ciruela P, Carmona G, Martínez A., et al. The epidemiology of invasive Streptococcus pneumoniae disease in Catalonia (Spain). A hospital-based study. Vaccine 2002; 20:2989-94.

14. Robinson KA, Baughman W, Rothrock G, Barrett NL, Pass M, Lexau C et al. Epidemiology of invasive Streptococcus pneumoniae infections in the United States, 1995-1998: Opportunities for prevention in the conjugate vaccine era. JAMA 2001; 285:1729-35

15. Whitney CG, Farley MM, Hadler J, Harrison LH, Bennett NM, Lynfield R, et al. Decline in invasive pneumococcal disease after the introduction of protein-polysaccharide conjugate vaccine. N Engl J Med 2003; 348:1737-45

16. Eskola J, Takala AK, Pekkanen E, Kalliokoski R, Leinonem M. Epidemiology of invasive pneumococcal infections in children in Finland. JAMA $1992 ; 268: 3323-27$.

17. Venetz I, Schopfer K, Mühlemann K. The Swiss Pneumococcal Study Group. Paedriatic invasive pneumococcal disease in Switzerland 1985-1994. Int J Epidemiol 1998; 27:1101-4.

18. Centers for Disease Control and Prevention. Preventing pneumococcal disease among infants and young children: Recommendations of the Advisory Committee on Immunization Practices (ACIP). MMWR 2000;49 (No. RR-9):1-35.

Rev Esp Salud Pública 2004, Vol. 78, N. ${ }^{\circ} 4$ 
19. Fedson DS \& Scott AG. The burden of pneumococcal disease among adults in developed and developing countries: what is and is not know. Vaccine 1999; 17:S11-9.

20. Fedson DS: The clinical effectiveness of pneumococcal vaccination: a brief review. Vaccine 1999; 17:S85-90.

21. Butler JC \& Schuant A. Epidemiology of pneumococcal infections in the elderly. Drugs Aging 1999; $15: 11-9$

22. Plouffe JF, Breiman RF, Facklam RR. Bacteremia with Streptococcus pneumoniae. JAMA 1996; 275:194-8.

23. Ortqvist A, Hedlund J, Grillner L, Jalonen E, Kallings I, Leinonen M, Kalin M. Aetiology, outcome and prognostic factors in community-acquired pneumoniae requiring hospitalisation. Eur Resp J 1990;3:1105-13.

24. Ramón de Juanes J, Gil A, González A, Arrazola P, Ansede JC. Hospitalizaciones por neumonía adquirida en la comunidad en dos hospitales generales. Enferm Infecc Microbiol Clin 2000; 18:243.

25. Salkinlampi V, Herva E, Haikala R, Liimatainen O, Renkonen OV, Leionen M. Epidemiology of invasive Streptococcus pneumoniae in adults in Finland. Epidemiol Infect 1997; 118:7-15.
26. Olivier C, Begne P, Cohen R, Floret D. Meningites à pneumocoque de l'enfant. Bull Epidemiol Hebdom 2000; 16:67-9.

27. Porath A, Schlaeffer F, Leiberman D. The epidemiology of community-adquired pneumonia among hospitalised adults. J Infect 1997; 34:41-8.

28. Zangwill KM, Vadheim CM, Vannier AM, Hemenway LS, Greenberg DP, Ward JI. Epidemiology of invasive pneumococcal disease in Southern California: Implications for the desing and conduct of a pneumococcal conjugate vaccine efficacy trial. J Infect Dis 1996; 174:752-9.

29. De Benoist AC, Laurent E, Goulet V. Infections invasives à Haemophilus influenzae, Listeria monocitogenes, méningoco que, pneumocoque, streptocoque groupe A et groupe B en France en 1997-evolution 1991-1997. Bull Epidemiol Hebdom 1999; 15:57-9.

30. Laurische H, Grimand O, Waight P, Johnson AP, George RC, Miller E. Pneumococcal bacteremia and meningitis in England and Wales, 1993 to 1995. Commun Dis Public Health 1998; 1:22-7.

31. Hausdorff WP, Siber G \& Paradiso PR. Geographical differences in invasive pneumococcal disease rates and serotype frequency in young children. Lancet 2001;357:950-2. 